\title{
Mucinous tubular and spindle cell carcinoma
}

\section{Joanna Rzymkowska', Magdalena Dudek², Marcin Ligaj³, Tomasz Kalinowski², Tomasz Demkow²}

${ }^{1} \mathrm{M}$. Skłodowska-Cuire Memorial Oncology Centre - Institute, Warsaw, Poland

2Urooncology Clinic, M. Skłodowska-Curie Memorial Oncology Institute, Warsaw, Poland

${ }^{3}$ Histopathology Department, M. Skłodowska-Curie Memorial Oncology Institute, Warsaw, Poland

\section{KEY WORDS}

mucinous tubular spindle renal carcinoma

\section{ABSTRACT}

A case of a 30-year-old female patient diagnosed with mucinous tubular and spindle cell carcinoma (MT\&SCC) of her left kidney of the basis of an abdominal ultrasound scan performed when nonspecific pain is presented. The histopathology report revealed a classic type of MT\&SCC. Photographs of histopathological samples are shown. Nephrectomy was applied as sole treatment. A follow-up of six years confirmed surgery to be a sufficient approach. The discussion presents a review of up to date world literature concerning the histopathological features, prognosis, and treatment of MT\&SCC.

\section{INTRODUCTION}

Mucinous tubular and spindle cell carcinoma (MT\&SCC) is a rare kidney cancer listed in the WHO classification since 2004. Treatment standards for this particular type of cancer have not yet been established. The necessity to differentiate this type of cancer with other types of kidney cancer has been previously stressed [1].

We present a case of a 30-year-old female patient who underwent nephroureterectomy due to a single large tumor of the left kidney.

\section{CASE REPORT}

A 30-year old woman reported with an abdominal ultrasound scan performed for nonspecific abdominal pain. The ultrasound revealed the presence of a $62 \times 52 \mathrm{~mm}$ spherical tumor located in the upper pole of the patient's left kidney. The lesion was solid, hyperechogenic, and well-demarcated. It showed no signs of infiltration of neighboring tissues, but did model the hilum. Within the tumor, a strongly hyperechogenic and a hypoechogenic section were found. No other abnormalities were noted.

A CT scan was performed - the tumor was originally assessed at 40 H.U. and reached 48 H.U. after contrast administration. No signs of disease dissemination were found.

The patient's physical examination, blood morphology, biochemical analysis results, and chest X-ray were normal, and she did not present with hematuria. Her GFR was measured by means of scintigraphy and was found to be $111 \mathrm{ml} / \mathrm{min}$ (left kidney $49 \%$, right kidney $51 \%$ of total), which was just below the age norm (115 ml/min).

A left nephroureterectomy was performed (in another center). No complications followed the procedure. The pathologist's report was as follows:

On gross examination a pale compact mass measuring $6 \times 5 \times$ $4.5 \mathrm{~cm}$ was seen towards one of the kidney poles. The mass was found to bulge the fibrous kidney capsule, without obvious capsule breach. No necrosis or hemorrhage was seen in the tumor tissue. The hilum area was not involved. The renal parenchyma beyond the mass appeared normal.

On microscopic examination, sections showed a triphasic tumor containing: 1) spindle-cell areas; 2) tubulo-papillary areas with focal myxoid, edematous stroma; and 3) compact areas of numerous "collapsed" tubules focally embedded in "bubbly" stroma. Tumor cells in all three components showed only minor nuclear atypia and vesicular nuclei with indistinct nucleoli seen only under 400x magnification (as in Fuhrman grade 2). Mitotic activity did not exceed two mitotic figures in 10 high-power fields. There was lymphocytic sprinkling throughout the tumor and focal aggregates of foamy macrophages were seen. There was no evidence of necrosis or angiolymphatic invasion. The tumor was found to infiltrate the fibrous capsule without capsule breach.

Immunostains and histochemistry revealed the tumor cells in all components to express: pan-cytokeratin (CK AE1/3), high-molecular weight cytokeratin (CK HMW [34BE12]), and vimentin. Cytokeratin 7 was only noted in the tubulo-papillary component. CD10 was negative.

In PAS/Alcian Blue staining the "bubbly" stroma was blue, consistent with mucus, and basal laminas of compact tubules were decorated red.

The main differential diagnosis included papillary RCC (pRCC), collecting duct carcinoma, and metanephric adenoma. Papillary RCC rarely shows extracellular mucin and lacks a spindle cell component, unless accompanied by a sarcomatoid change (in which case the spindle cell component shows high-grade features). CD10 antigen is frequently seen in $\mathrm{PRCC}$, but it was negative in our case. Collecting duct carcinoma is an aggressive tumor showing highgrade histology. Metanephric adenoma shows tubulo-papillary architecture, but stromal and spindle cell components are lacking and cytokeratin 7 is usually negative.

The diagnosis of MT\&SCC PT1b was established.

A postoperative CT scan and annual abdominal ultrasound scans showed no signs of recurrence. The patient required no additional treatment and remains under observation. During 56 months of follow-up no signs of dissemination were noted, and she has successfully given birth to a healthy child.

\section{DISCUSSION}

MT\&SCC has been documented to develop at various ages, $33-80$ years $[1-4 ; 6,7,8]$. Shen et al. report a ratio of male:female of $1: 3$ [8].

Nonspecific abdominal pains are usually reported, and patients may present with hematuria. $[3,4,5,11]$ This was also true of this patient, who has developed a large mass of tumor before any symptoms were noted.

Although MT\&SCC has only recently been isolated from RCCs, shown to be a distinct form of cancer usually consisting of elongated, small tubules with cuboidal cells, or cords of spindled cells showing mild atypia, variant forms have already been described. $[2,3 ; 5,-9]$ It does not express kidney-specific cadherin (Ksp-cad), 


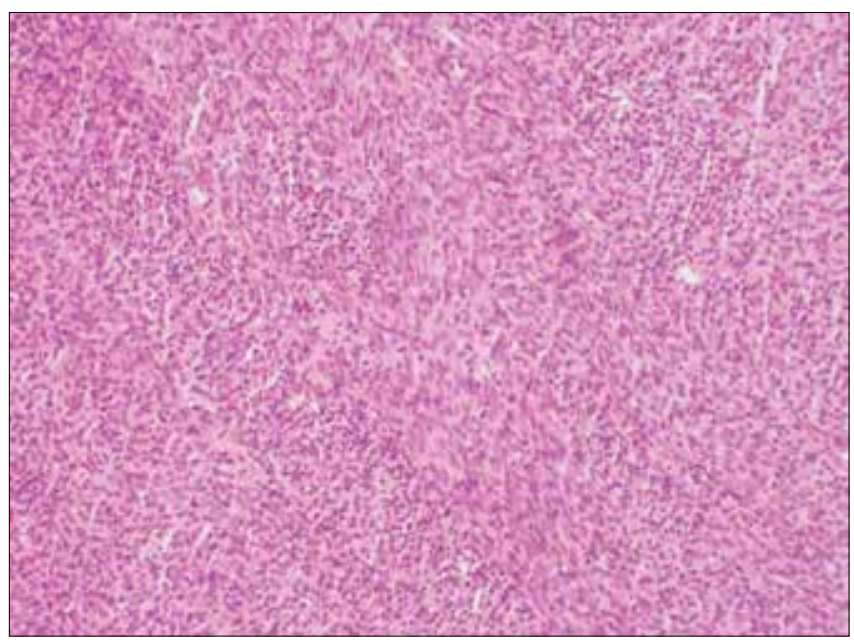

Fig. 1. Spindle cell component of the tumor (x40 magnification), characteristic of MT\&SCC.

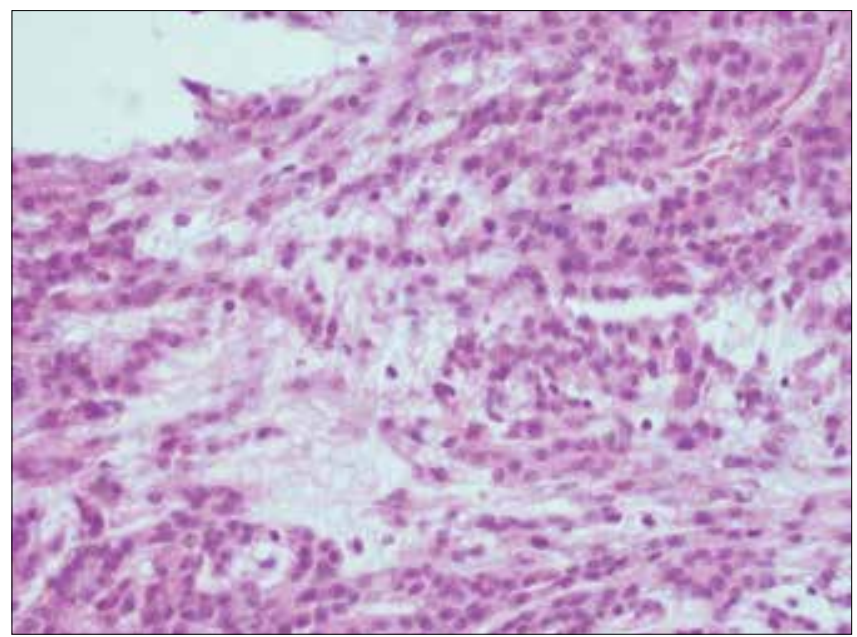

Fig. 3. "Bubbly" tumor stroma (x200 magnification), the third distinguishing feature of MT\&SCC.

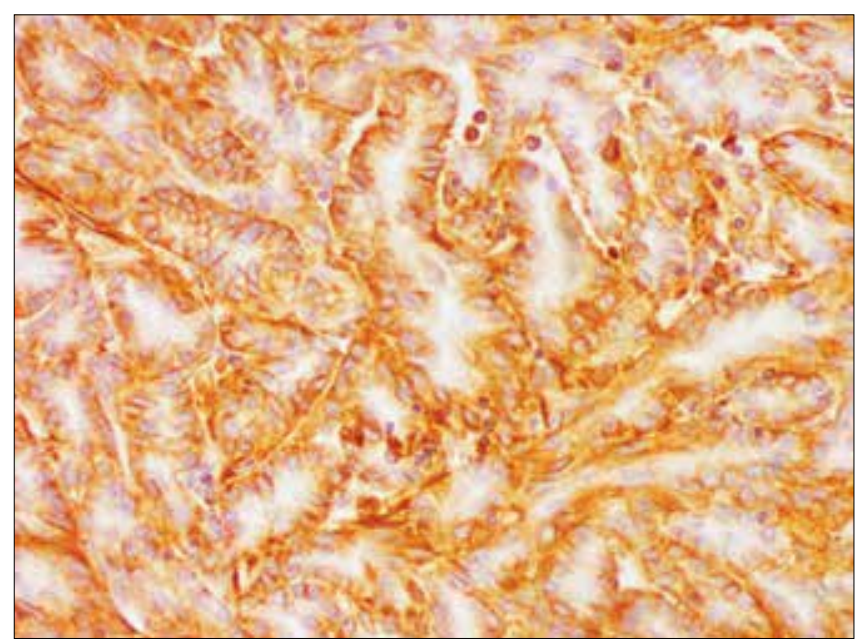

Fig. 5. Pan-cytokeratin expressed (CK AE1/3) in the tubulo-papillary component (x200 magnification).

typical of the ascending loop of Henle cells [10]. Ours is a case of a "classic" MT\&SCC.

Immunohistochemistry has a limited role in differential diagnosis of MT\&SCC and pRCC. Both tumors show several analogies in morphology

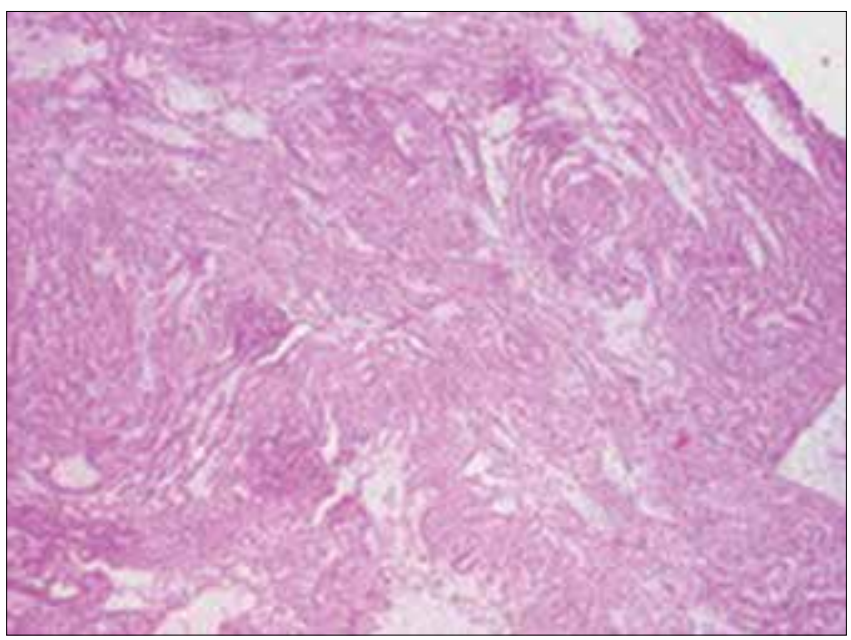

Fig. 2. Tubulo-papillary tumor component (X40 magnification), the second characteristic feature of MT\&SCC.

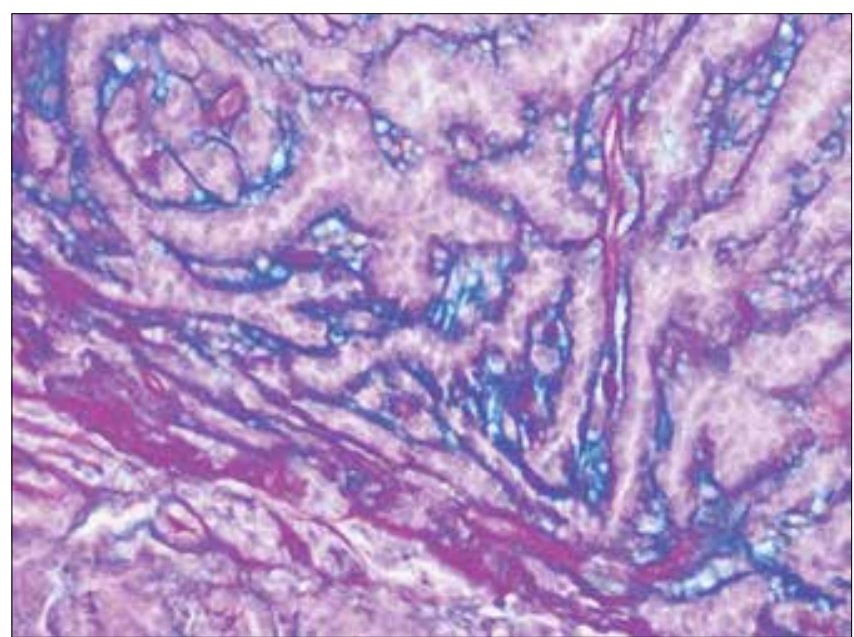

Fig. 4. PAS/Alcian Blue staining reveals the blue decoration of the stroma, characteristic of mucus producing neoplasms (x200 magnification).

and immunoprofile [10] and some authors regarded them as variants of the same entity [12]. Main differences can be found at the level of genetic mutations typical of MT\&SCC and pRCC $[13,14]$, but we did not perform cytogenetic or molecular analysis of the presented case.

As in our case, MT\&SCC rarely shows signs of dissemination $[8,11]$.

A treatment standard for this particular type of cancer has not yet been established.

MT\&SCC seems to be associated with good prognosis, unless sarcomatoid lesions are present within the neoplasmatic tissue [1, $5,6,7]$. Patients with sarcomatoid and disseminated disease have much worse prognosis than those with the classic type, even when large tumors confined to the kidney are discovered [1-8]. The main treatment method up till now remains radical nephrectomy, even in cases of disseminated disease $[7,11]$. Usually they do not require adjuvant treatment $[1,11]$.

Our patient was treated with nephroureterectomy, which up till now seems to have proved sufficient.

\section{CONCLUSIONS}

MT\&SCC is associated with good prognosis and surgical treatment usually proves sufficient. Careful histopathological examination remains the key to diagnosis and further treatment. 
As more evidence is gathered, a standard for the treatment should be established.

\section{REFERENCES}

1. Kato $\mathrm{M}$, Soga $\mathrm{N}$, Arima $\mathrm{K}$, Sugimura Y: A case of renal mucinous tubular and spindle cell carcinoma. Int J Urol 2009; 16 (8): 699-701.

2. Farghaly $\mathrm{H}$ : Mucin poor mucinous tubular and spindle cell carcinoma of the kidney, with nonclassic morphologic variant of spindle cell predominance and psammomatous calcification. Ann Diagn Pathol 2012; 16 (1): 59-62.

3. Driss $\mathrm{M}$, Doghri $\mathrm{R}, \mathrm{Mrad} \mathrm{K}$, et al: An unusual renal neoplasm: mucinous tubular and spindle cell carcinoma. Prog Urol 2008; 18 (4): 253-255.

4. Geramizadeh G, Salehipour M, Moradi A: Mucinous tubular and spindle cell carcinoma of kidney: a rare case report and review of the literature. Indian J Pathol Microbiol 2009; 53 (4): 514-516.

5. Otani M, Shimizu T, Serizawa H, Nagashima Y: Mucinous tubular and spindle cell carcinoma of the kidney: Report of a case with imprint cytologic features. Acta Cytol 2006; 50 (6): 680-682.

6. Pillay N, Ramdial PK, Cooper K, Batuule D: Mucinous tubular and spindle cell carcinoma with aggressive histomorphology - a sarcomatoid variant. Hum Pathol 2008; 39 (6): 966-969.

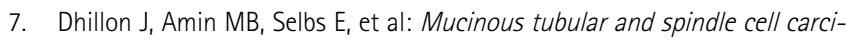
noma of the kidney with sarcomatoid change. Am J Surg Pathol 2009; 33 (1): 44-49.

8. Shen SS, Ro JY, Tamboli P, et al: Mucinous tubular and spindle cell carcinoma of kidney is probably a variant of papillary renal cell carcinoma with spindle cell features. Ann Diagn Pathol 2007; 11 (1): 13-21

9. Paner GP, Srigley JR, Radhakrishnan A, et at: Immunohistochemical analysis of mucinous tubular and spindle cell carcinoma and papillary renal cell carcinoma of the kidney: significant immunophenotypic overlap warrants diagnostic caution. Am J Surg Pathol 2006; 30 (1): 13-19.
10. Kuehn A, Paner GP, Skinnider BF: Expression analysis of kidney-specific cadherin in a wide spectrum of traditional and newly recognized renal epithelial neoplasms: diagnostic and histogenetic implications. Am J Surg Pathol 2007; 31 (10): 1528-1533.

11. Trabelesi A, Stita W, Yacoubi MT, et al: Renal mucinous tubular and spindle cell carcinoma. Can Urol Assoc J 2008; 2 (6): 635-636.

\section{Correspondence}

Joanna Rzymkowska

M. Skłodowska-Cuire Memorial

Oncology Centre - Institute

5, Roentgena Street

02-781 Warsaw, Poland

phone: +48546021085

rzymekropka@gmail.com 